\title{
Reliability and validity of a computerized questionnaire to measure fat intake in Belgium
}

\author{
Corneel Vandelanotte ${ }^{\mathrm{a}, *}$, Christophe Matthys ${ }^{\mathrm{b}}$, Ilse De Bourdeaudhuij ${ }^{\mathrm{a}}$ \\ ${ }^{\mathrm{a}}$ Faculty of Medicine and Health Sciences, Department of Movement and Sport Sciences, \\ Ghent University, Watersportlaan 2, 9000 Ghent, Belgium \\ ${ }^{\mathrm{b}}$ Faculty of Medicine and Health Sciences, Department of Public Health, Ghent University, Watersportlaan 2, \\ 9000 Ghent, Belgium
}

Received 1 October 2003; accepted 15 December 2003

\begin{abstract}
This study evaluated the reliability and validity of a Belgian computerized questionnaire to measure fat intake. A total of 86 adults completed a 48 -item computerized fat intake questionnaire, subsequently kept a 7-day diet record, and finally completed the questionnaire again. For validity, a Pearson correlation of 0.67 for total fat intake and of 0.60 for percent energy from fat were found between the computerized questionnaire and the diet records. Gross misclassification between the two methods was $5.8 \%$ for total fat intake and for percent energy from fat. Cohen's $\kappa$ values were respectively 0.27 and 0.29 . For reliability, intraclass correlation coefficients ranged from 0.70 to 0.87 between the two administrations of the computerized fat intake questionnaire. Gross misclassification between the administrations was $1.2 \%$ for total fat intake and for percent energy from fat, $\kappa$ values were respectively 0.64 and 0.50 . These results indicate that this fat intake questionnaire has an acceptable reliability and validity. (C) 2004 Elsevier Inc. All rights reserved.
\end{abstract}

Keywords: Diet records; Fat intake; Food frequency questionnaire; Computer; Validation; reliability

\section{Introduction}

The Belgian population has an average fat intake of about $42 \%$ energy from fat, which is well above the recommended $30 \%$ energy from fat [1-3]. This places most of these

* Corresponding author. Tel.: 32.(0)9.264.63.23; fax: 32.(0)9.264.64.84.

E-mail address: Corneel.Vandelanotte@UGent.be (C. Vandelanotte, Ph.D. student) 
individuals higher risk for developing chronic illnesses, such as coronary heart disease and cancer [4-6]. Fat intake interventions are needed and food consumption assessments are necessary to evaluate the effects of these programs. When working with large sample sizes, food frequency questionnaires (FFQs) seem to be the most appropriate method for collection of nutrition data, because they are low in cost and can reach a wide range of ages and target groups [7]. Computerized assessment of FFQs can further increase these advantages, as data can be automatically stored on file, reducing hours of data entry as well as reducing coding errors and the risk of lost data [8]. They can also be programmed to eliminate missing data, requiring participants to answer all questions, and skip-patterns can be used to avoid superfluous questions (e.g., for vegetarians) [8,9].

A fat intake questionnaire specifically designed for the Belgian dietary habits has not previously been developed. In the present study the reliability and validity of a computerized questionnaire to measure fat intake in Belgian individuals was evaluated. The questionnaire was not designed to assess a complete diet history, but was developed to give personalized fat intake feedback to participants in a nutrition education program. The relative validity of this questionnaire was examined in relation to a 7-day estimated diet record. The reproducibility of the questionnaire is assessed by means of a test-retest procedure.

\section{Methods and materials}

\subsection{Computerized fat intake questionnaire}

The computerized fat intake questionnaire consists of 48 questions divided into 7 categories of food items (Table 1) and takes about 20 minutes to administer. The computerized self-assessment is very simple, allowing individuals with no computer literacy to obtain their fat score. The questionnaire was developed at the Ghent University in cooperation with the Flemish Institute for Health promotion (VIG). The selection of the food items was done by experienced dieticians. All food items contributing to fat intake in the usual Belgian diet were included in the questionnaire [10]. Participants were asked how frequently they consumed the food products during a usual day, week, or month. Each question was guided by several examples of the food product, mostly including brand names, and an average portion size. For each question a coefficient was calculated based on average fat content $[10,11]$, average portion size [12], and time span on the questionnaire (day, week, or month) of the food product. This coefficient was multiplied with the frequency of consumption and summed for all questions, leading to an individual total fat intake score, expressed in average grams of fat per day.

Apart from the fat intake questions some demographic characteristics were also assessed, i.e., sex, age, weight, length, education, level of physical activity, and household size. Finally, participants were asked whether they were vegetarian, cooked dinner themselves, and did household shopping themselves. Individual recommended energy intake was calculated from standard recommended energy intake tables based on height, weight, sex, age, and activity level [1]. This allowed expression of reported fat intake on the computerized questionnaire as a percentage of total energy intake, with the assumption that the respon- 
Table 1

Computerized fat intake questionnaire*

\begin{tabular}{|c|c|}
\hline Food Group & Item \\
\hline \multirow{8}{*}{$\begin{array}{l}\text { 1. Bread and } \\
\text { cereals }\end{array}$} & 1. How many slices of bread do you eat a day? \\
\hline & 2. How many biscuits or crackers do you eat a week? \\
\hline & 3. How many French loaves do you eat a week? \\
\hline & 4. How many sandwiches do you eat a week? \\
\hline & 5. How many rolls with chocolate do you eat a week? \\
\hline & 6. How many croissants or rolls with fruit do you eat a week? \\
\hline & 7. How many bowls of muesli do you eat a week? \\
\hline & 8. How many bowls of muesli with chocolate and fruit (cruesli) do you eat a week? \\
\hline \multirow{10}{*}{$\begin{array}{l}\text { 2. Spreads, } \\
\text { fillings, and } \\
\text { butter }\end{array}$} & 9. How many slices or portions of fat cheese do you eat a week? \\
\hline & 10. How many slices or portions of half-fat cheese do you eat a week? \\
\hline & 11. How many slices or portions of low-fat cheese do you eat a week? \\
\hline & 12. How many slices or portions of fat meat products do you eat a week? \\
\hline & 13. How many slices or portions of half-fat meat products do you eat a week? \\
\hline & 14. How many slices or portions of low-fat meat products do you eat a week? \\
\hline & 15. How many slices of bread with chocolate spread do you eat a week? \\
\hline & 16. How many slices of bread with full-fat butter or margarine do you eat a week? \\
\hline & 17. How many slices of bread with half-fat margarine do you eat a week? \\
\hline & 18. How many slices of bread with low-fat margarine do you eat a week? \\
\hline \multirow{3}{*}{$\begin{array}{l}\text { 3. Milk and } \\
\text { milk products }\end{array}$} & 19. How many glasses of full milk products do you eat a week? \\
\hline & 20. How many glasses of semi-skimmed milk products do you eat a week? \\
\hline & 21. How many glasses of low-fat milk products do you eat a week? \\
\hline \multirow{6}{*}{$\begin{array}{l}\text { 4. Prepared } \\
\text { meals }\end{array}$} & 22. How many pizzas do you eat a month? \\
\hline & 23. How many lasagnes do you eat a month? \\
\hline & 24. How many pastas Bolognaise do you eat a month? \\
\hline & 25. How many croquets (mashed potatoes) do you eat a month? \\
\hline & 26. How many servings of mashed or baked potatoes do you eat a month? \\
\hline & 27. How many portions of potato chips do you eat a month? \\
\hline \multirow{7}{*}{$\begin{array}{l}\text { 5. Meat, fish, } \\
\text { and eggs }\end{array}$} & 28. How many portions of fat fish do you eat a month? \\
\hline & 29. How many portions of low-fat fish do you eat a month? \\
\hline & 30. How many portions of high-fat meat do you eat a week? \\
\hline & 31. How many portions of half-fat meat do you eat a week? \\
\hline & 32. How many portions of low-fat meat do you eat a week? \\
\hline & 33. How many portions of vegetarian replacement products do you eat a week? \\
\hline & 34. How many eggs do you eat a week? \\
\hline \multirow{6}{*}{$\begin{array}{l}\text { 6. Sauce, gravy, } \\
\text { and dressing }\end{array}$} & 35. How many tablespoons of mayonnaise do you eat a week? \\
\hline & 36. How many tablespoons of other mayonnaise like sauces do you eat a week? \\
\hline & 37. How many tablespoons of dressing and cream do you eat a week? \\
\hline & 38. How many tablespoons of gravy do you eat a week? \\
\hline & 39. How many tablespoons of thinned gravy do you eat a week? \\
\hline & 40. How many tablespoons of cheese sauce do you eat a week? \\
\hline \multirow{8}{*}{$\begin{array}{l}\text { 7. Snacks, cake } \\
\text { and biscuits }\end{array}$} & 41. How many slices of cake and waffles do you eat a week? \\
\hline & 42. How many slices of pie do you eat a week? \\
\hline & 43. How many dry biscuits do you eat a week? \\
\hline & 44. How many biscuits with muesli, chocolate, vanilla, nuts, ... do you eat a week? \\
\hline & 45. How many chocolate candy bars do you eat a week? \\
\hline & 46. How many portions of crisps and nuts do you eat a week? \\
\hline & 47. How many portions of ice cream do you eat a month? \\
\hline & 48. How many portions of cheese and meat e.g. at receptions do yo \\
\hline
\end{tabular}

* Examples of food products, brand names, and portion sizes that guided these questions on the questionnaires are not shown. 
dents' energy intake and expenditure was in balance. The questionnaire was pilot tested among four experts and four individuals out of the potential target group. The computer questionnaire showed a good readability and understandability and was easier to administer than the paper-and-pencil version, even by participants with no computer literacy.

\subsection{Reference instrument}

A 7-day estimated diet record was used as a reference instrument to validate the computerized fat intake questionnaire. This method does not depend on memory, is generally accepted as a relatively valid and reliable food consumption method, and has been used before in validation studies [13-16]. Together with a detailed instruction form and a standardized example of a recording, participants were given a set of prestructured forms for recording the diet for 7 days. They were asked to record all food and beverages consumed, including brand names, methods of food preparation, and ingredients or recipes of mixed dishes. They also had to describe portions sizes (in household measures, e.g., cups of coffee, spoons of sugar) and had to indicate the point in time of consumption (at breakfast, in the morning, at supper, in the afternoon, at dinner, in the evening). Participants were asked to monitor their 'habitual' food intake and to avoid any changes in diet. It was explained to them that changing their diet during monitoring would result in "wrong" advice. The record forms were inspected for completeness by dieticians and ambiguous records were resolved by asking questions of participants via telephone. The diet records were analysed using the Becel Nutrition software which calculated a mean daily individual total fat intake in grams [17]. The software used the same food composition tables and the same manual for determining portion sizes as were used in the computerized fat intake questionnaire.

\subsection{Participants and procedures}

Participants, aged 22-61 years, were recruited at random in and around the city of Ghent (Belgium). For each participant there were two contacts, either at the university or at participants' homes (using a portable computer). At the first contact participants completed the computerized fat intake questionnaire and were given detailed oral instruction about the diet recording, a diet recording instruction form and the 7-day diet record forms. Participants also signed an informed consent statement approved by the Ghent University Ethics Committee. Diet recording started the day after for 7 consecutive days. The second contact was immediately after the recording period. Participants returned the diet record forms and completed the questionnaire once more. As compensation, expert dietary advice was given by the dieticians to participants who complied with the study protocol.

\subsection{Statistical methods}

For validity, paired sample $t$ tests were used to examine differences between the means of the computerized fat intake questionnaire and the 7-day diary. Pearson product-moment correlation coefficients were used to examine the agreement between the computerized questionnaire and the diet records. Spearman rank-order correlation coefficients were also 
Table 2

Means and standard deviations (SD) for participants' age, height, weight, body mass index, recommended energy intake, fat intake, and percentage energy from fat

\begin{tabular}{|c|c|c|c|c|c|c|}
\hline \multirow[t]{2}{*}{ Characteristic } & \multicolumn{2}{|c|}{$\begin{array}{l}\text { Total Sample } \\
(N=86)\end{array}$} & \multicolumn{2}{|c|}{$\begin{array}{l}\text { Men } \\
(N=45)\end{array}$} & \multicolumn{2}{|c|}{$\begin{array}{l}\text { Women } \\
(N=41)\end{array}$} \\
\hline & Mean & SD & Mean & SD & Mean & SD \\
\hline Age $(y)$ & 35.1 & 12.1 & 35.3 & 13.1 & 34.7 & 11.1 \\
\hline Height (m) & 1.74 & 0.1 & 1.81 & 0.1 & 1.67 & 0.1 \\
\hline Weight (kg) & 71.2 & 11.6 & 78.6 & 10.2 & 63.4 & 6.9 \\
\hline Body mass index $\left(\mathrm{kg} / \mathrm{m}^{2}\right)$ & 23.3 & 2.8 & 23.8 & 2.4 & 22.6 & 3.0 \\
\hline Recommended energy intake (kcal) & 2659 & 579.5 & 3108 & 472.1 & 2181 & 100.7 \\
\hline \multicolumn{7}{|l|}{ Computerized questionnaire at contact 1} \\
\hline Total fat intake $(\mathrm{g})$ & 99.9 & 33.4 & 118.2 & 30.7 & 80.5 & 24.0 \\
\hline Energy from fat $(\%)$ & 34.2 & 10.4 & 34.9 & 9.9 & 33.4 & 10.4 \\
\hline \multicolumn{7}{|l|}{ Computerized questionnaire at contact 2} \\
\hline Total fat intake $(\mathrm{g})$ & 97.7 & 37.6 & 113.3 & 34.6 & 74.6 & 22.2 \\
\hline Energy from fat $(\%)$ & 33.0 & 10.3 & 33.3 & 10.0 & 30.7 & 9.2 \\
\hline \multicolumn{7}{|l|}{ 7-Day diet records } \\
\hline Total fat intake (g) & 97.9 & 34.1 & 111.0 & 33.4 & 82.0 & 27.4 \\
\hline Energy from fat $(\%)$ & 33.1 & 10.2 & 32.1 & 8.7 & 33.9 & 11.5 \\
\hline
\end{tabular}

computed but not reported, as they were very similar to the Pearson correlation. Skewed data were normalized using natural logarithmic transformations. To evaluate the ability of the questionnaire to classify individuals correctly according to broad categories of fat intake, subjects were classified into three categories of fat intake (tertiles) separately by each method. Cohen's $\kappa$ was used to assess the degree of agreement in classification between the two methods. Gross misclassification beyond an adjacent tertile was also calculated.

For test-retest reliability, single-measure intraclass correlation coefficients (ICC) were used between the computerized fat intake questionnaire at contacts 1 and 2, and added with Cohen's $\kappa$ and gross misclassification.

All analyses were performed using SPSS version 11.0 software (SPSS, Inc., Chicago, IL). Statistical significance was set at an $\alpha$ level of 0.05 .

\section{Results}

A total of 109 participants volunteered for this study. Of these, six dropped out during data gathering (because of time constraints or lack of motivation); five had very high fat intake measures on the computerized questionnaire (mean $\pm 2 \mathrm{SD}$ ) and were classified as outliers with extreme values; and 12 returned diet records of useless quality, leaving 86 participants (45 men and 41 women) who complied with all requirements. The mean age for the overall group was 35 years; the mean BMI was 23.8 for men and 22.6 for women; and the recommended energy intake for the total group was $2656 \mathrm{kcal}$ (Table 2).

Mean total fat intake according to the computerized fat intake questionnaire at contact 1 was $99.9 \pm 33.4 \mathrm{~g} / \mathrm{day}$, representing $34.2 \%$ of the total energy from fat (Table 2). At contact 2 , fat intake was $97.7 \pm 37.6 \mathrm{~g} /$ day, representing $33 \%$ of the total energy from fat. Mean fat 
Table 3

Pearson correlations between the computerized fat intake questionnaire and the 7-day diet records for fat intake and percent energy from fat

\begin{tabular}{lll}
\hline Characteristic & Fat Intake $(\mathrm{g})$ & $\%$ Energy From Fat \\
\hline Total group $(n=86)$ & $0.67^{\ddagger}$ & $0.60^{\ddagger}$ \\
Sex & & \\
$\quad$ Men $(n=45)$ & $0.42^{\ddagger}$ & $0.49^{\ddagger}$ \\
$\quad$ Women $(n=41)$ & $0.68^{\ddagger}$ & $0.71^{\ddagger}$ \\
Age & & \\
$\quad<40$ years $(n=61)$ & $0.73^{\ddagger}$ & $0.64^{\ddagger}$ \\
$\quad>40$ years $(n=25)$ & $0.38^{\S}$ & $0.42^{\S}$ \\
Education & $0.72^{\ddagger}$ & $0.63^{\ddagger}$ \\
$\quad$ Lower $(n=34)^{*}$ & $0.63^{\ddagger}$ & $0.57^{\ddagger}$ \\
$\quad$ Higher $(n=52)$ & & \\
Body mass index & $0.66^{\ddagger}$ & $0.59^{\ddagger}$ \\
$\quad<25(n=68)$ & $0.70^{\ddagger}$ & $0.61^{\ddagger}$ \\
$\quad>25(n=18)$ & & \\
Level of physical activity & & $0.64^{\ddagger}$ \\
$\quad$ Low $(n=42)$ & $0.69^{\ddagger}$ & $0.60^{\ddagger}$ \\
$\quad$ Medium $(n=32)$ & $0.73^{\ddagger}$ & $0.41^{\S}$ \\
$\quad$ High $(n=12)$ & $0.26^{\S}$ & $0.66^{\ddagger}$ \\
Cooking for household & & $0.50^{\ddagger}$ \\
$\quad$ Regularly $(n=53)$ & $0.69^{\ddagger}$ & \\
$\quad$ Not regularly $(n=33)$ & $0.64^{\ddagger}$ & $0.67^{\ddagger}$ \\
Shopping for household & & $0.25^{\S}$ \\
$\quad$ Regularly $(n=63)$ & $0.73^{\ddagger}$ & \\
$\quad$ Not regularly $(n=23)$ & $0.31^{\S}$ &
\end{tabular}

* Lower education $=$ education until age 18 years, no higher education or college.

${ }^{\dagger}$ Low physical activity $=$ administrative job, employee, $\ldots$ with no sports in leisure time; medium physical activity $=$ job in maintenance, selling, light to moderate physical efforts, . . . with some sports in leisure time; high physical activity $=$ job in agriculture, forestry, vigorous manual labor, $>2$ hours of sports per day.

${ }^{*} P<0.01$.

${ }^{\S} P<0.05$.

intake according to the diet records was $97.9 \pm 34.1 \mathrm{~g} /$ day, and percent energy from fat according to these records was $33.1 \%$.

For validity, there were no significant differences between means for total fat intake and for percent energy from fat between the computerized fat intake questionnaire and 7-day diet records.

Table 3 shows Pearson correlations between the computerized fat intake questionnaire and the 7-day diet records. For total fat intake a correlation of 0.67 was found. A slightly lower correlation from 0.60 was found for percent energy from fat.

Correlations were also assessed for different subgroups, although it must be realized that some subgroups were small. Higher correlations for total fat intake and for percent energy from fat intake were found for women, for participants $<40$ years of age, and for participants who did regular household shopping as compared to their counterparts. Remarkably lower correlations were found for participants with a high level of physical activity and participants who did not engage in regular household shopping as compared to their counterparts.

For total fat intake gross misclassification between the two methods was $5.8 \%$ (total 
Table 4

Classification in tertiles of the computerized fat intake questionnaire compared to classification in tertiles of the 7-day diet records for fat intake and percent energy from fat; gross misclassification and Cohen's $\kappa$

\begin{tabular}{|c|c|c|c|c|c|c|c|c|c|c|c|}
\hline \multirow{2}{*}{$\begin{array}{l}\text { Tertile on Diet } \\
\text { Record }\end{array}$} & \multicolumn{3}{|c|}{$\begin{array}{l}\text { Lowest Tertile on Fat } \\
\text { Intake Questionnaire }\end{array}$} & \multicolumn{3}{|c|}{$\begin{array}{l}\text { Middle Tertile on Fat } \\
\text { Intake Questionnaire }\end{array}$} & \multicolumn{3}{|c|}{$\begin{array}{l}\text { Highest Tertile on Fat } \\
\text { Intake Questionnaire }\end{array}$} & \multirow{2}{*}{$\begin{array}{l}\text { Gross } \\
\text { Misclassi- } \\
\text { fication }\end{array}$} & \multirow[t]{2}{*}{$\begin{array}{l}\text { Cohen's } \\
\kappa\end{array}$} \\
\hline & Lov & & le Highest & Lowest & Middle & Highest & Lowest & Middle & Highest & & \\
\hline \multicolumn{12}{|l|}{ Fat intake (g) } \\
\hline Total group & 18 & 9 & 2 & 9 & 10 & 8 & 3 & 11 & 16 & $5.8 \%$ & 0.27 \\
\hline Male & 4 & 1 & 2 & 4 & 4 & 5 & 3 & 7 & 15 & $11.1 \%$ & 0.20 \\
\hline Female & 14 & 8 & 0 & 5 & 6 & 3 & 0 & 4 & 1 & $0.0 \%$ & 0.17 \\
\hline \multicolumn{12}{|c|}{$\%$ Energy from fat } \\
\hline Total group & 16 & 9 & 3 & 11 & 11 & 7 & 2 & 9 & 18 & $5.8 \%$ & 0.29 \\
\hline Male & 8 & 6 & 2 & 5 & 3 & 4 & 1 & 8 & 8 & $6.6 \%$ & 0.14 \\
\hline Female & 8 & 3 & 1 & 6 & 8 & 3 & 1 & 1 & 10 & $4.9 \%$ & 0.45 \\
\hline
\end{tabular}

group), Cohen's $\kappa$ was 0.27 (Table 4). Remarkably lower gross misclassification was found for women $(0 \%)$ as compared to men $(11.1 \%)$. For percent energy from fat, the gross misclassification was again $5.8 \%$ (total group), and Cohen's $\kappa$ was 0.29 . Higher $\kappa$ values were found for women $(0.45)$ as compared to men $(0.14)$.

For reliability, Table 5 presents the single measure intraclass correlation coefficients (ICC), expressed in grams of fat intake, for the two computerized fat intake questionnaire administrations at contacts 1 and 2 . These correlations ranged from ICC $=0.70$ to ICC $=$ 0.87. The ICC for total fat intake was 0.86 , and 0.81 for percent energy from fat. Lowest ICC was for milk and milk products (0.70). Gross misclassification between the two administrations was $1.2 \%$ for total fat intake and percent energy from fat (total group), $\kappa$ values were respectively 0.64 and 0.50 (Table 6).

\section{Discussion}

If behavioral measurements such as fat intake are measured within a health promotion or educational context, it is very important that the validity and reliability of these assessment

Table 5

Single measure intraclass correlations (ICC) for the computerized fat intake questionnaire between contacts 1 and 2

\begin{tabular}{lr}
\hline & ICC \\
\hline Fat intake (g) & 0.86 \\
$\%$ Energy from fat (\%) & 0.81 \\
Bread and cereals (g) & 0.85 \\
Spreads, fillings and butter (g) & 0.83 \\
Milk and milk products (g) & 0.70 \\
Prepared meals (g) & 0.87 \\
Meat, fish, and eggs (g) & 0.76 \\
Sauce, gravy, and dressing (g) & 0.74 \\
Snacks, cake, and biscuits (g) & 0.87 \\
\hline
\end{tabular}


Table 6

Classification in tertiles of the computerized fat intake questionnaire at contact 1 compared to that at contact 2 for fat intake and percent energy from fat; gross misclassification and Cohen's $\kappa$

\begin{tabular}{|c|c|c|c|c|c|c|c|c|c|c|c|}
\hline \multirow{2}{*}{$\begin{array}{l}\text { Tertiles at } \\
\text { Contact } 2\end{array}$} & \multicolumn{3}{|c|}{$\begin{array}{l}\text { Lowest Tertile at } \\
\text { Contact } 1\end{array}$} & \multicolumn{3}{|c|}{$\begin{array}{l}\text { Middle Tertile at } \\
\text { Contact } 1\end{array}$} & \multicolumn{3}{|c|}{$\begin{array}{l}\text { Highest Tertitle at } \\
\text { Contact } 1\end{array}$} & \multirow{2}{*}{$\begin{array}{l}\text { Gross } \\
\text { Misclassi- } \\
\text { fication }\end{array}$} & \multirow{2}{*}{$\begin{array}{l}\text { Cohen's } \\
\kappa\end{array}$} \\
\hline & Lowest & Middle & Highest & Lowest & Middle & Highest & Lowest & Middle & Highest & & \\
\hline \multicolumn{12}{|l|}{ Fat intake (g) } \\
\hline Total group & 23 & 5 & 1 & 5 & 20 & 4 & 1 & 4 & 23 & $1.2 \%$ & 0.64 \\
\hline Male & 4 & 2 & 1 & 1 & 10 & 3 & 0 & 3 & 21 & $2.2 \%$ & 0.59 \\
\hline Female & 19 & 3 & 0 & 4 & 10 & 1 & 1 & 1 & 2 & $0.0 \%$ & 0.56 \\
\hline \multicolumn{12}{|c|}{$\%$ Energy from fat } \\
\hline Total group & 24 & 6 & 0 & 5 & 15 & 8 & 1 & 9 & 18 & $1.2 \%$ & 0.50 \\
\hline Male & 13 & 5 & 0 & 2 & 5 & 4 & 0 & 7 & 10 & $0.0 \%$ & 0.42 \\
\hline Female & 11 & 1 & 0 & 3 & 10 & 4 & 1 & 2 & 8 & $2.4 \%$ & 0.59 \\
\hline
\end{tabular}

tools is guaranteed. In the present study the reliability and validity of a computerized questionnaire to measure fat intake was evaluated in relation to a 7-day diet record. To assess the validity of a new dietary assessment tool it is recommended that several different validation techniques be used simultaneously [18], as there is still an ongoing debate over how validity should correctly be assessed [19]. In this study four general accepted methods are presented $[9,20]$ : comparison of means, correlation analysis, cross-classification, and use of $\kappa$ statistics.

On basis of means comparison it could be stated that the computerized fat intake questionnaire can estimate the mean intakes for total fat intake and for percent energy from fat at the group level, inasmuch as no significant differences were found.

Several authors reported that correlations between an FFQ and diet records $>0.5$ support the validity of the FFQ $[9,20-22]$. In the present study a correlation of 0.67 was found for total fat intake. From this, the computerized fat intake questionnaire can be considered to be a valid assessment tool. In comparison with the review of validation studies of fat intake questionnaires with $<50$ questions by van Assema et al. [15], the correlations found in this study are generally higher.

Correlations between the computerized fat intake questionnaire and the diet records for "percentage energy" from fat were somewhat lower (0.60); however, when compared to the findings of van Assema et al. [15], they are still better than in most of the reported studies.

The majority of studies do not report separate correlations for subgroups. As mentioned earlier, these results should be interpreted with caution, as some subgroups were small. Higher correlations for female participants were found, which is consistent with other studies $[9,15]$. A possible explanation is that women are more accurate in reporting diet because of greater responsibility for household cooking or shopping [15]. This could apply in this study, as $78 \%$ and $89 \%$ of the women reported respectively cooking or shopping regularly, as compared to $48 \%$ and $63 \%$ of men (data not shown). This explanation can also be confirmed by the finding that correlations for participants who do regular household shopping and cooking are respectively much higher $(0.73$ vs 0.31$)$ and slightly higher $(0.69$ vs 0.64$)$ compared to those who do not. As in the study by van Assema et al. [15], we found higher 
correlations for participants $<40$ years of age and participants with less education. We agree with van Assema et al. [15] that lower or higher correlations in some subgroups might be explained by the eating patterns of these subgroup that correspond more or less with the food items that were measured on the questionnaire.

Masson et al. [9] stated that $\kappa$ statistics are meaningful for presenting associations with percentages of cross-classification. These investigators also reported that in dietary validation studies more than $50 \%$ of participants must be correctly classified into tertiles, and that no more than $10 \%$ of participants should be grossly misclassified to be acceptable. $\kappa$ Values between 0.61 and 0.80 are described as indication of good agreement, between 0.41 and 0.60 as moderate agreement, between 0.21 and 0.40 as fair agreement, and below 0.20 as poor agreement [23]. In this study we found that for total fat intake $51 \%$ of participants were correctly classified and for percent energy from fat $52 \%$ of participants. For fat intake and for percent energy from fat $5.8 \%$ of participants were grossly misclassified. These results further support the validity of the computerized fat intake questionnaire and are in line with other validation studies $[9,15,24,25]$. The $\kappa$ statistics, however, showed only fair agreement for total fat (0.27) and for percent energy from fat (0.29). Acceptable agreement was only found in women for percent energy from fat (0.45). Several other validation studies also have difficulties finding acceptable $\kappa$ values $>0.4[9,15,24]$. This is in line with the findings of Portney and Watkins [26], who state that this statistic is usually an underestimate of true reliability if used for continuous data, as in our study. The higher $\kappa$ value for women can probably be explained in the same way as the higher correlations for women described earlier.

When measuring the reliability of a questionnaire, intraclass correlation coefficients (ICC) from 0.75 or higher are considered good to very good [8]. For total fat intake we found 0.86 and for percent energy from fat 0.81 , indicating excellent reliability. This finding is strengthened by a very low gross misclassification and $\kappa$ values showing good agreement. According to Cade et al. [9], $\kappa$ statistics in conjunction with correlation coefficients are good measures to express reliability. Reliability is often not reported when evaluating a new dietary assessment tool; however, comparable results were found in a study from van Assema et al. [15].

This study has several limitations. The diet record method is considered as an important reference method for questionnaire validation. However, because the present study had a cross-sectional design, it remains unclear whether our computerized questionnaire is suitable to detect changes in fat intake over time. It is also a limitation that our questionnaire cannot estimate total energy intake without an unacceptable increase of questions. Finally, estimated food records were used as well as standard portion sizes for the computer questionnaire. The choice for estimated versus weighed portions to decrease the burden for the respondents could have resulted in less precise fat estimates in the present study.

In conclusion, several statistical methods have indicated that our newly developed and computerized fat intake assessment tool has an acceptable reliability and validity.

\section{Acknowledgments}

C. Vandelanotte, who is the main author of this manuscript gathered all data, conducted the analysis and wrote the manuscript. C. Matthys gave advice on validating food frequency 
questionnaires and on statistics. I. De Bourdeaudhuij contributed in developing test protocols, provided statistical advice and is the principal investigator of the health promotion unit of the Department of Movement and Sport Sciences. All authors contributed to the final writing of the manuscript. This study was financially supported by the Ghent University and the Flemish Fund for Scientific Research. The authors also thank dietician Erika Vanhauwaert from the Flemish Institute for Health promotion (VIG) for cooperation in developing the questionnaire, Nathalie Vanloocke for assistance in data gathering, and dieticians An D'Hooge and Annelot Vasseur for analyzing the diet records.

\section{References}

[1] National Council on Nutrition (Nationale raad voor de voeding). Dietary recommendations for Belgium (Voedingsaanbevelingen voor België). Brussels: Ministry of Social Affairs, Public Health and Environment, 1997.

[2] Staessen L, De Bacquer D, De Henauw S, De Backer G, Van Peteghem C. Fatty acid composition of the Belgian diet: estimates derived from the Belgian interuniversity research on nutrition and health. Ann Nutr Metab 1998;42:151-9.

[3] De Henauw S, De Backer G. Nutrient and food intakes in selected subgroups of Belgian adults. Br J Nutr 1999;81(Suppl 2):S37-42.

[4] Staessen L, DeHenauw S, DeBacquer D, DeBacker G, VanPeteghem C. Consumption of fatty acids in Belgium and its relationship with cancer mortality. Cancer Lett 1997;114:109-11.

[5] Zyriax BC, Windler E. Dietary fat in the prevention of cardiovascular disease-a review. Eur J Lipid Sci Technol 2000;102:355-65.

[6] Hooper L, Summerbell CD, Higgins JPT, Thompson RL, Capps NE, Smith GD, Riemersma RA, Ebrahim S. Dietary fat intake and prevention of cardiovascular disease: systematic review. Br Med J 2001;322:75763.

[7] Persson PG, Carlsson S, Grill V, Hagman U, Lundgren AC, Ostenson CG, Perers M, Wallen A. Food frequency questionnaire versus 7-day weighed dietary record information on dietary fibre and fat intake in middle-aged Swedish men. Scand J Soc Med 1998;26:75-80.

[8] Steiner DL, Norman GR. Health measurement scales: a practical guide to their development and use. Oxford: Oxford University Press, 1995.

[9] Cade J, Thompson R, Burley V, Warm D. Development, validation and utilisation of food-frequency questionnaires-a review. Public Health Nutr 2002;5:567-87.

[10] Belgian Food Composition Table, 3th ed (Nubel). Brussels: Ministry of Public Health, 1999.

[11] Dutch Food Composition Table 1996 (Nevo). Den Haag: Stichting NEVO, 1998.

[12] National Council on Health (Hoge Gezondheidsraad). Measures and weights, a manual for standardized and quantified food products in Belgium (Maten en gewichten). Brussels: Ministry of Social Affairs, Public Health and Environment, 2001.

[13] Bingham SA, Cassidy A, Cole TJ, Welch A, Runswick SA, Black AE, Thurnham D, Bates C, Khaw KT, Key TJA, Day NE. Validation of weighed records and other methods of dietary assessment using the 24-h urine nitrogen technique and other biological markers. Br J Nutr 1995;73:531-50.

[14] Bingham SA, Gill C, Welch A, Day K, Cassidy A, Khaw KT, Sneyd MJ, Key TJA, Roe L, Day NE. Comparison of dietary assessment methods in nutritional epidemiology-weighed records vs. 24-H recalls, food-frequency questionnaires and estimated-diet records. Br J Nutr 1994;72:619-43.

[15] van Assema P, Brug J, Ronda G, Steenhuis I. The relative validity of a short Dutch questionnaire as a means to categorize adults and adolescents to total and saturated fat intake. J Hum Nutr Dietet 2001;14:377-90.

[16] Lee KY, Uchida K, Shirota T, Kono S. Validity of a self-administered food frequency questionnaire against 7-day dietary records in four seasons. J Nutr Sci Vitam 2002;48:467-76.

[17] Becel Nutrition Program. Rotterdam: Univlever Co., 1992. 
[18] Nelson M. The validation of dietary assessment. In: Margetts BM, Nelson M, editors. Design concepts in nutritional epidemiology. Oxford: Oxford University Press, 1996. p 241-72.

[19] de groot C, van Staveren WA, Riboli E. Abstracts on the Third International Conference on Dietary Assement Methods. Eur J Clin Nutr 1998;52:51-89.

[20] Masson LF, McNeill G, Tomany JO, Simpson JA, Peace HS, Wei L, Grubb DA, Bolton-Smith C. Statistical approaches for assessing the relative validity of a food-frequency questionnaire: use of correlation coefficients and the kappa statistic. Public Health Nutr 2003;6:313-21.

[21] Plesko M, Cotugna N, Aljadir L. Usefulness of a brief fruit and vegetable FFQ in a college population. Am J Health Behav 2000;24:201-8.

[22] Brunner E, Stallone D, Juneja M, Bingham S, Marmot M. Dietary assessment in Whitehall II: comparison of $7 \mathrm{~d}$ diet diary and food-frequency questionnaire and validity against biomarkers. Br J Nutr 2001;86: 405-14.

[23] Altman DG. Statistical-analysis of comparisons between laboratory methods. J Pathol 1991;44:700-1.

[24] van Assema P, Brug J, Kok G, Brants H. The reliability and validity of a Dutch questionnaire on fat consumption as a means to rank subjects according to individual fat intake. Eur J Cancer Prev 1992;1: $375-80$.

[25] Rohrmann S, Klein G. Validation of a short questionnaire to qualitatively assess the intake of total fat, saturated, monounsaturated, polyunsaturated fatty acids, and cholesterol. J Hum Nutr Diet 2003;16:111-7.

[26] Portney LG, Watkins MP. Foundations of clinical research. Norwalk, CT: Appleton \& Lange, 1993. 